Ciencia y Salud, Vol. V, No. 2, mayo-agosto, 2021 •ISSN (impreso): 2613-8816 • ISSN (en línea): 2613-8824

DOI: https://doi.org/10.22206/cysa.2021.v5i2.pp139-148

\title{
EFECTIVIDAD DEL TEST DE SANGRE OCULTA EN HECES POR INMUNOHISTOQUÍMICA PARA EL TAMIZAJE DE LESIONES COLÓNICAS: SERIE DE CASOS
}

\section{"Effectiveness of the fecal immunochemical test (FIT) for Colonic Injury Screening: Case Series}

\section{Lucía Bayona, Ricardo Acra-Tolari, Carla Mendoza Valiente y Michelle Estrella Vargas}

Recibido: 3 de abril, $2020 \bullet$ Aprobado: 6 de julio, 2020

Cómo citar: Bayona L, Acra-Tolari R, Mendoza Valiente C, Estrella Vargas M. Efectividad del test de sangre oculta en heces por inmunohistoquímica para el tamizaje de lesiones colónicas: serie de casos. cysa [Internet]. 10 de junio de 2021 [citado 16 de junio de 2021];5(2):139-48. Disponible en: https://revistas.intec.edu.do/index.php/cisa/article/view/2217

\section{Resumen}

Introducción: el cáncer colorrectal (CCR) es el tercer cáncer con mayor incidencia en hombres y en mujeres en la República Dominicana. Se ha encontrado una elevada incidencia entre la presencia sangre oculta en heces $(\mathrm{SOH})$ y la expresión de $\mathrm{CCR}$, al igual que para la de pólipos colorrectales. El propósito de este estudio fue identificar datos tangibles que demostraran el uso de esta prueba como herramienta costo-efectiva capaz de detectar lesiones premalignas de CCR.

Metodología: se realizó un estudio observacional-descriptivo, serie de casos, dentro del Hospital General Plaza de la Salud, donde se analizaron pacientes con una prueba de sangre oculta en heces por inmunohistoquímica positiva (SOHi), que luego se realizaron una colonoscopia dentro del periodo de enero 2016 a febrero 2020.

\footnotetext{
a Médico Gastroenteróloga. Gerente del departamento de gastroenterología del Hospital General Plaza de la Salud. Investigadora Principal ORCID: 0000-0003-4915-0766, Correo-e: ybayona@hgps.org.do

b Voluntario de investigación del Hospital General Plaza de la Salud. Universidad Iberoamericana (UNIBE). ORCID: 0000-0002-9979-420,

Correo-e: ricardo_Acra@hotmail.com
}

\begin{abstract}
Introduction: Colorectal cancer (CRC) is the third cancer with the highest incidence in men and women in the Dominican Republic. A high incidence has been found between the presence of occult blood in stool and the expression of CRC, as well as for colorectal polyps. The purpose of this study was to identify tangible data that will demonstrate the use of this test as a cost-effective tool, capable of detecting premalignant CRC lesions.

Methodology: An observational-descriptive, series of cases study was carried out within the Hospital General Plaza de la Salud, where patients were analyzed with a fecal immunochemical test (FIT), followed by a colonoscopy within the period of January 2016 to February 2020.

Results: 378 patients were found with a positive FIT and a subsequent colonoscopy, of these, 90 patients didn't present

\footnotetext{
b Universidad Iberoamericana UNIBE. ORCID: 0000-0002-5844-6895, Correo-e: Carlamendoza97@gmail.com
}

${ }^{\mathrm{c}}$ Universidad Iberoamericana UNIBE. ORCID: 0000-0002-1448-7767, Correo-e: michiestrella@hotmail.com
\end{abstract}


Resultados: se encontraron 378 con pruebas de SOHi positivas, que luego se realizaron colonoscopia, de estos, 90 no presentaron hallazgos patológicos, 128 presentaron pólipos y 160 pacientes presentaron otros tipos de hallazgos patológicos en las colonoscopias.

Conclusión: la prueba de SOHi representa una herramienta costo-efectiva para la detección temprana y prevención de lesiones colorrectales malignas, y recomendamos su uso dentro del tamizaje temprano del cáncer colorrectal.

Palabras clave: inmunohistoquímica; colonoscopia; turbidimétrico; aglutinación; In vitro

\section{Introducción}

Según el Observatorio Global de Cáncer, en el 2018, el cáncer colorrectal (CCR) a nivel mundial fue el tercer cáncer más común. De la misma manera, en República Dominicana representó el tercer cáncer más común en ambos sexos, así como la tercera causa de muerte por cáncer en hombres y la cuarta en mujeres ${ }^{1}$.

La prueba de sangre oculta en heces (SOH) es una prueba de laboratorio que se usa para verificar muestras de heces en busca de sangre oculta ${ }^{2}$. En el 2016, en un estudio realizado por Irma Martín Álvarez et al. se encontró una elevada incidencia entre la presencia sangre oculta en heces y la expresión de CCR. Por otro lado, los resultados positivos de esta prueba también se han relacionado con la presencia de pólipos colorrectales debido a pequeños sangrados intermitentes provenientes de los mismos. ${ }^{3,4}$

Otro estudio, en el 2019, también determinó que la concentración de hemoglobina en heces mediante pruebas de sangre oculta en heces por inmunohistoquímica (SOHi), funciona como predictor de riesgo para adenomas avanzados y cáncer colorrectal, por lo que se recomienda dentro de su tamizaje, seguido de una colonoscopia en los casos positivos ${ }^{5,6}$. pathological findings, 128 had polyps, and 160 had other types of pathological findings in colonoscopies.

Conclusion: The FITs represents a cost-effective tool for screening and prevention of malignant colorectal lesions, and we recommend its use within early colorectal cancer screening.

Keywords: Immunochemistry; Colonoscopy; Turbidimetric principle; Agglutination; In vitro.

Los pólipos colorrectales se definen como crecimientos anormales del revestimiento del colon y el recto, estos tienen diversas clasificaciones dependiendo de su morfología, características histológicas y comportamiento, donde la mayor atención que se les dedica a sus características es debido a su poder de malignizar ${ }^{7}$. Normalmente estos se presentan de forma asintomática, sin embargo, en ocasiones pueden derramar pequeñas cantidades de sangre, las cuales pueden ser detectadas mediante pruebas de $\mathrm{SOH}^{8}$.

La prueba de $\mathrm{SOH}$ seguida por una colonoscopia en los casos positivos, es la estrategia de elección para el tamizaje poblacional de cáncer colorrectal en la mayoría de los países europeos. En poblaciones de riesgo medio, se recomienda la detección de sangre oculta en heces cada 1 o 2 años, por su inocuidad y bajo costo ${ }^{9,10}$.

Existen dos tipos de pruebas de $\mathrm{SOH}$ : la prueba de Guayaco y la prueba por inmunohistoquímica. La prueba de $\mathrm{SOH}$ tipo guayaco utiliza una actividad similar a la enzima peroxidasa, que se encuentra en la hemoglobina, para generar una reacción oxidativa con un reactivo y producir un color azul. Debido a que otras sustancias tienen actividad peroxidasa o pseudoperoxidasa, estas pueden provocar una reacción de falso positivo si están presentes en las heces 
(carnes rojas, algunas frutas y verduras), también algunos medicamentos de uso común, como la aspirina y los medicamentos antiinflamatorios no esteroideos (AINEs), pueden causar hemorragia oculta; por lo tanto, estos y otros medicamentos son irritantes gástricos y deben evitarse antes de realizar esta prueba. La prueba inmunohistoquímica usa una proteína especializada o anticuerpo que se adhiere a la hemoglobina ${ }^{11,12}$.

La comparación de los métodos de $\mathrm{SOH}$ de tipo Guayaco e inmunohistoquímica es de larga data. Ejemplo de ello es el estudio realizado por Guittet L. et al., en el año 2007, donde compararon los resultados de estas dos pruebas y declaran estar a favor de la sustitución de la prueba de Guayaco por la prueba inmunohistoquímica, debido a que beneficiaría a los pacientes que están en mayor riesgo de padecer adenomas colónicos ${ }^{13}$.

En un estudio realizado en el 2012 se determinó que la prueba $\mathrm{SOHi}$ tiene diversas ventajas sobre la prueba de Guayaco para la detección temprana del cáncer colorrectal. Debido a su alta sensibilidad y especificidad $y$, entre otras ventajas, este método se ha convertido en uno de los más utilizados ${ }^{14,15}$.

M. Ángeles Gutiérrez-Stampa et al., en el 2019, analizaron la utilidad de la prueba $\mathrm{SOHi}$ en pacientes sintomáticos en la unidad de atención primaria, donde concluyeron que la misma tiene una alta eficacia diagnóstica en la detección del $\mathrm{CCR}^{16}$. De forma similar, Valle Llufrio et al. determinaron, que el método de $\mathrm{SOHi}$ es de gran importancia en el diagnóstico temprano de lesiones de colon, por lo que debe realizarse a todo paciente mayor a 50 años ${ }^{17}$.

Otros estudios a favor de la prueba de SOHi sobre la de Guayaco afirman que la prueba de inmunohistoquímica es más selectiva y es menos afectada por factores no patológicos (como dieta o consumo de drogas), y que debe ser incluida en las estrategias de personalización del tamizaje en personas con test de Guayaco negativo para reducir el riesgo de $\mathrm{CCR}^{18,19}$.

El propósito de este estudio fue identificar datos tangibles que demostraran el uso de esta prueba como herramienta costo-efectiva capaz de detectar lesiones premalignas de CCR. El tener presente que esta prueba permite una detección temprana de este tipo de cáncer, debe llevarla a ser tomada en cuenta como analítica de rutina en pacientes con factores de riesgo para el mismo.

\section{Material y métodos}

Esta investigación es de tipo observacional-descriptiva, serie de casos, con datos retrospectivos y transversales donde se analizaron pacientes que se realizaron una prueba de SOHi dentro del periodo de enero del 2016 a febrero del 2020.

La muestra fue de 378 pacientes, quienes presentaron resultados positivos en el test de $\mathrm{SOHi}$, y que luego se realizaron una colonoscopia. Se tomaron en cuenta pacientes de todas la edades y sexo. Al momento de recolectar los datos de la muestra, se agruparon los pacientes en tres grupos: "Pacientes con pólipos", "Pacientes con otras patologías" y "Pacientes sin hallazgos patológicos".

En el grupo "Pacientes con pólipos" se incluyeron aquellos que presentaron alguna clase de pólipo, a excepción de los que mostraron adenomas avanzados con desarrollo de malignidad, o los que tenían historia de colectomía. Estos se incluyeron en el grupo de "Pacientes con otras patologías", junto a los que presentaron otros hallazgos patológicos (divertículos, hemorroides, carcinomas, etc.).

Se excluyeron los pacientes cuyas colonoscopias fueron realizadas previas a la prueba de SOHi. En el caso del paciente haberse realizado dos o más pruebas en el periodo de tiempo seleccionado, se escogió la más próxima a la fecha de la colonoscopia y se descartaron las demás. No se tomaron más criterios de exclusión. 
El método de la prueba de sangre oculta en heces utilizado fue el iFOB, el cual es un test inmunológico basado en el principio turbidimétrico por aglutinación de látex, para la determinación cuantitativa in vitro de hemoglobina humana en heces. Los reactivos contienen partículas de látex de poliestireno, cubiertas con anticuerpos IgG de cabra anti-hemoglobina humana, en un tampón de $20 \mathrm{mmol} / \mathrm{L}$. La hemoglobina del paciente presente en el tubo recolector, reacciona con las partículas de látex recubiertas, causando una aglutinación que puede ser medida a través de un fotómetro. El grado de turbidez es proporcional a la concentración de la hemoglobina en la muestra.

Las recomendaciones dictadas por el hospital para la realización de esta prueba fueron: evitar tomar la muestra en ciertas condiciones que proveen sangrado tales como hemorroides, trabajo dental, estreñimiento, estudio de tacto rectal, durante o después de tres días del período menstrual, presencia de sangre en la orina, así como también, evitar el consumo de alcohol y aspirina por al menos 48 horas antes de la prueba.

Los reportes de colonoscopias fueron tomados de la base de datos electrónica del hospital, al igual que los reportes de patología. Los pólipos se clasificaron en adenomatosos, hiperplásicos, tubulares, tubulovellosos, vellosos, inflamatorios, y en el caso haberse reportado más de un pólipo se clasificó como "Dos o más tipos de pólipos".

Se excluyó del estudio cualquier dato con el que se pueda identificar la identidad de los pacientes con el motivo de proteger su confidencialidad.

Para el análisis y manejo de los datos se utilizaron los programas Excel y SPSS.

\section{Resultados}

De un total de 16,271 pacientes con prueba de $\mathrm{SOH}$, 1,619 obtuvieron resultados positivos, de los cuales, 378 se realizaron una colonoscopia subsecuente a la prueba. De estos, 90 no presentaron hallazgos patológicos, 128 presentaron pólipos y 160 pacientes presentaron otros tipos de hallazgos patológicos.

Se determinaron los tipos de pólipos más frecuentes: 43 adenomatosos (34\%), 29 hiperplásicos (23\%), 28 tubulares $(22 \%), 16$ con dos o más tipos de pólipos (12\%), 6 tubulovellosos (5\%), 3 vellosos $(2 \%)$ e 3 inflamatorios (2\%). Las principales localizaciones de estos fueron: en el recto (21\%), en el colon descendente $(20 \%)$, en el colon ascendente (19\%), en el colon sigmoides (14\%), en el colon transverso $(10 \%)$ en el ciego (8\%), en el ángulo hepático (3\%), en recto-sigmoides (3\%), en la válvula ileocecal (1\%) y en el ángulo esplénico (1\%). 
Efectividad del test de sangre oculta en heces por inmunohistoquímica para el tamizaje de lesiones colónicas: serie de casos

Tabla 1. Clasificación de hallazgos patológicos por grupos etarios

\begin{tabular}{|c|c|c|c|c|c|c|}
\hline \multicolumn{7}{|c|}{ Case Processing Summary } \\
\hline & \multicolumn{6}{|c|}{ Cases } \\
\hline & \multicolumn{2}{|c|}{ Valid } & \multicolumn{2}{|c|}{ Missing } & \multicolumn{2}{|c|}{ Total } \\
\hline & $\mathrm{N}$ & Percent & $\mathrm{N}$ & Percent & $\mathrm{N}$ & Percent \\
\hline $\begin{array}{l}\text { GRUPO ETARIO * } \\
\text { Diagnostico }\end{array}$ & 378 & $100.0 \%$ & 0 & $0.0 \%$ & 378 & $100.0 \%$ \\
\hline
\end{tabular}

\begin{tabular}{|c|c|c|c|c|c|}
\hline & & & \multicolumn{3}{|c|}{ Diagnóstico } \\
\hline & & & PÓLIPO & $\begin{array}{c}\text { OTRA } \\
\text { PATOLOGÍA }\end{array}$ & $\begin{array}{l}\text { ESTUDIO } \\
\text { NORMAL }\end{array}$ \\
\hline \multirow[t]{10}{*}{ GRUPO ETARIO } & \multirow[t]{2}{*}{$0-18$ AÑOS } & Recuento & 1 & 2 & 0 \\
\hline & & \% GRUPO ETARIO & $33.3 \%$ & $66.7 \%$ & $0.0 \%$ \\
\hline & \multirow[t]{2}{*}{ 19-44 AÑOS } & Recuento & 13 & 40 & 36 \\
\hline & & $\%$ GRUPO ETARIO & $14.6 \%$ & $44.9 \%$ & $40.4 \%$ \\
\hline & \multirow[t]{2}{*}{ 45-64 AÑOS } & Recuento & 67 & 76 & 53 \\
\hline & & $\%$ GRUPO ETARIO & $34.2 \%$ & $38.8 \%$ & $27.0 \%$ \\
\hline & \multirow[t]{2}{*}{ 65-84 AÑOS } & Recuento & 43 & 41 & 0 \\
\hline & & $\%$ GRUPO ETARIO & $51.2 \%$ & $48.8 \%$ & $0.0 \%$ \\
\hline & \multirow[t]{2}{*}{$>85$ AÑOS } & Recuento & 4 & 1 & 1 \\
\hline & & $\%$ GRUPO ETARIO & $66.7 \%$ & $16.7 \%$ & $16.7 \%$ \\
\hline \multirow{2}{*}{\multicolumn{2}{|c|}{ Total }} & Recuento & 128 & 160 & 90 \\
\hline & & $\%$ GRUPO ETARIO & $33.9 \%$ & $42.3 \%$ & $23.8 \%$ \\
\hline
\end{tabular}

\begin{tabular}{|c|c|c|c|}
\hline & & & Total \\
\hline \multirow[t]{10}{*}{ GRUPO ETARIO } & \multirow[t]{2}{*}{ 0-18 AÑOS } & Recuento & 3 \\
\hline & & \% GRUPO ETARIO & $100.0 \%$ \\
\hline & \multirow[t]{2}{*}{ 19-44 AÑoS } & Recuento & 89 \\
\hline & & \% GRUPO ETARIO & $100.0 \%$ \\
\hline & \multirow[t]{2}{*}{ 45-64 AÑOS } & Recuento & 196 \\
\hline & & \% GRUPO ETARIO & $100.0 \%$ \\
\hline & \multirow[t]{2}{*}{ 65-84 AÑOS } & Recuento & 84 \\
\hline & & \% GRUPO ETARIO & $100.0 \%$ \\
\hline & \multirow[t]{2}{*}{$>85$ AÑOS } & Recuento & 6 \\
\hline & & $\%$ GRUPO ETARIO & $100.0 \%$ \\
\hline \multirow{2}{*}{\multicolumn{2}{|c|}{ Total }} & Recuento & 378 \\
\hline & & $\%$ GRUPO ETARIO & $100.0 \%$ \\
\hline
\end{tabular}

Fuente: análisis de la base de datos del estudio.

Tabla 2. Hallazgos patológicos según el sexo

Crosstab

\begin{tabular}{|c|c|c|c|c|c|}
\hline & & & \multicolumn{2}{|c|}{ SEXO } & \multirow[b]{2}{*}{ Total } \\
\hline & & & FEMENINA & MASCULINO & \\
\hline \multirow[t]{6}{*}{ Diagnostico } & \multirow[t]{2}{*}{ PÓLIPO } & Recuento & 62 & 66 & 128 \\
\hline & & $\%$ SEXO & $34.6 \%$ & $33.2 \%$ & $33.9 \%$ \\
\hline & \multirow[t]{2}{*}{ OTRA PATOLOGÍA } & Recuento & 68 & 92 & 160 \\
\hline & & $\%$ SEXO & $38.0 \%$ & $46.2 \%$ & $42.3 \%$ \\
\hline & \multirow[t]{2}{*}{ ESTUDIO NORMAL } & Recuento & 49 & 41 & 90 \\
\hline & & $\%$ SEXO & $27.4 \%$ & $20.6 \%$ & $23.8 \%$ \\
\hline \multirow{2}{*}{\multicolumn{2}{|c|}{ Total }} & Recuento & 179 & 199 & 378 \\
\hline & & $\%$ SEXO & $100.0 \%$ & $100.0 \%$ & $100.0 \%$ \\
\hline
\end{tabular}

Fuente: análisis de la base de datos del estudio. 
Tabla 3. Tipos de pólipos más frecuentes según el grupo etario

Tipo de Pólipo

Inflamatorio

Velloso

Tubulovelloso

Dos o más tipos de pólipos

Tubular

Hiperplásico

Adenomatoso
Edad

$0-18$ AÑOS

19-44 AÑOS

45-64 AÑOS

65-84 AÑOS

$>85$ AÑOS

Total

0-18 AÑOS

19-44 AÑOS

45-64 AÑOS

65-84 AÑOS

$>85$ AÑOS

Total

0-18 AÑOS

19-44 AÑOS

45-64 AÑOS

65-84 AÑOS

$>85$ AÑOS

Total

0-18 AÑOS

19-44 AÑOS

45-64 AÑOS

65-84 AÑOS

$>85$ AÑOS

Total

0-18 AÑOS

19-44 AÑOS

45-64 AÑOS

65-84 AÑOS

$>85$ AÑOS

Total

0-18 AÑOS

19-44 AÑOS

45-64 AÑOS

65-84 AÑOS

$>85$ AÑOS

Total

0-18 AÑOS

19-44 AÑOS

45-64 AÑOS

65-84 AÑOS

$>85$ AÑOS

Total
Frecuencia

0

1

2

0

0

3

0

0

1

2

0

3

0

0

4

4

2

0

6

0

0

9

9

7

0

16

0

2

16

10

0

28

0

7

15

5

2

29

1

3

20

17

2

43
Porcentaje

33.333

66.667

0

0

100

0

0

33.333

66.667

0

100

0

\section{0}

66.667

33.333

0

100

0

\section{0}

56.25

43.75

0

100

7.143

57.143

35.714

0

100

0

24.138

51.724

17.241

6.897

100

2.326

6.977

46.512

39.535

4.651

100

Fuente: análisis de la base de datos del estudio. 


\section{Gráfico 1. Pacientes con pólipos clasificados según el sexo dependiendo del grupo etario}

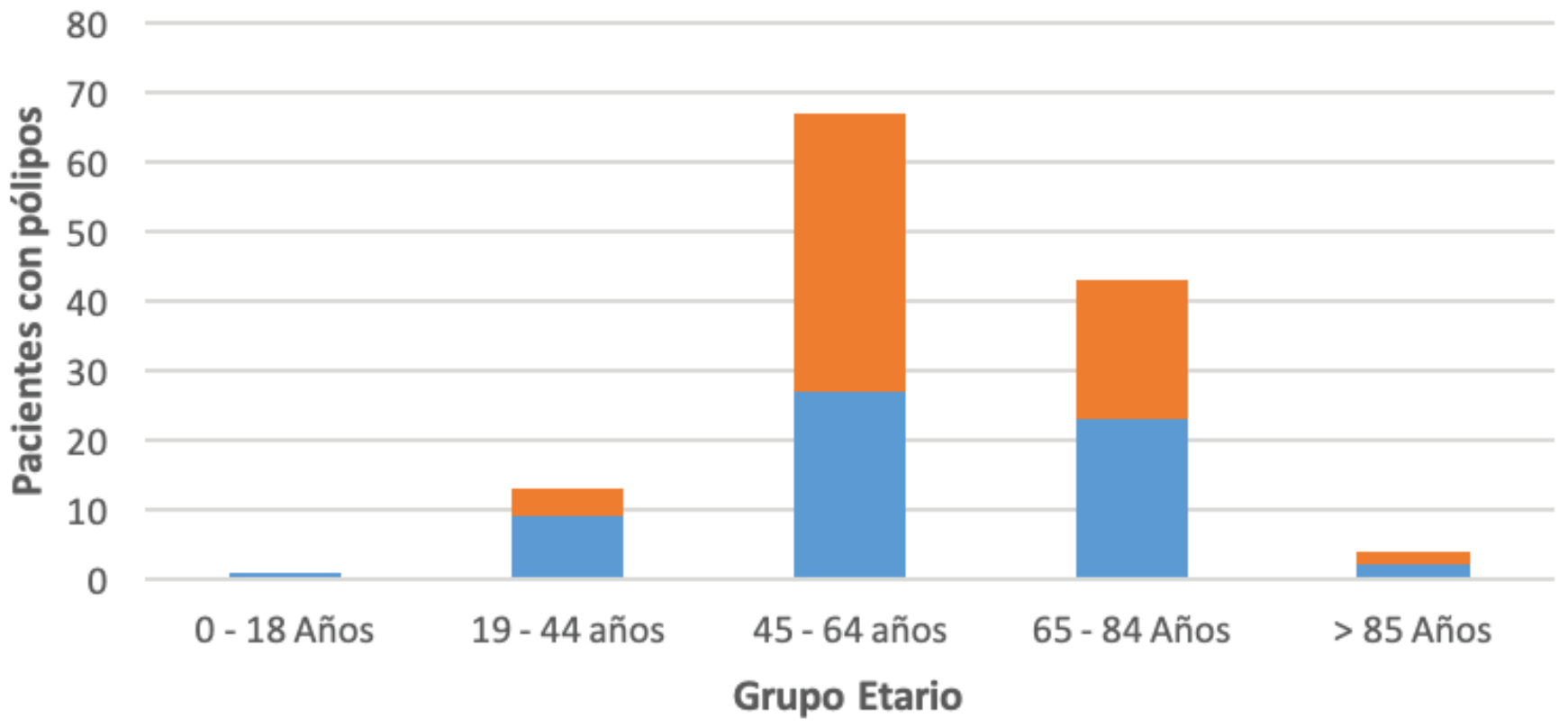

Fuente: análisis de la base de datos del estudio.

\section{Discusión}

Los pólipos colorrectales se presentan en aproximadamente el 25-30\% de los hombres y mujeres de cualquier etnia, mayores de 50 años. No todos los pólipos se convertirán en cáncer, y pueden pasar ańos antes de que un pólipo se convierta en malignidad. Existen diferentes factores de riesgo que aumentan las probabilidades de padecer estos, como son el tabaquismo, el consumo excesivo de alcohol, la obesidad, entre otros. ${ }^{20,21}$

En este estudio el tipo de pólipo más común fue el adenomatoso, coincidiendo con lo descrito por la universidad de Michigan ${ }^{21}$. La mayoría de los pólipos que llegan a malignizarse suelen ser adenomatosos, sin embargo, tienen un bajo porcentaje de convertirse en cáncer. Este proceso puede tardar muchos años, y con un buen tamizaje, el CCR derivado de estos es altamente prevenible.

Por otro lado, los pólipos de tipo velloso y tubulovelloso tienen una gran tendencia a malignizarse, pero, al igual que los adenomatosos, con un buen sistema de tamizaje, donde recomendamos que esté incluido la prueba de $\mathrm{SOHi}$, esto se puede evitar.

La aparición de pólipos tiende a ser más común en hombres que en mujeres ${ }^{22}$. Este estudio refuerza esta afirmación al mostrar una mayor incidencia de pólipos en el sexo masculino. Sin embargo, es de importancia resaltar que solo hubo cuatro pacientes de sexo masculino más que del sexo femenino.

Otra característica demográfica relevante para el estudio es que el riesgo para desarrollar adenomas 
colorrectales se duplica entre las edades de 50 a 74 años ${ }^{22}$. En este estudio, el grupo etario en el cual se observó mayor cantidad de pólipos fue el de 45 a 64 años de edad. Cabe destacar, que en este grupo etario se puede apreciar una mayor diferencia entre el número de casos por sexo, donde 40 pacientes eran masculinos y 27 femeninas.

\section{Conclusión}

En este estudio, más de una tercera parte de los pacientes que tenían una prueba de $\mathrm{SOHi}$ positiva, que posteriormente se realizaron una colonoscopia, presentaron algún tipo de pólipo. Cabe resaltar que existen diversas condiciones que pueden ocasionar la presencia de sangre oculta en heces, las cuales no fueron enfocadas en esta investigación, como es la presencia de malignidad, colectomía previa, hemorroides, diverticulosis, entre otros.

Los resultados de este estudio indican que la prueba de SOHi podría funcionar como una herramienta costo-efectiva para la detección temprana de lesiones premalignas. Especialmente si en los casos positivos se indica una colonoscopia. Por ello, es importante que esta prueba se tome en cuenta en los métodos de tamizaje para el CCR.

\section{Agradecimientos}

Nos gustaría agradecer al departamento de Laboratorio Clínico del Hospital General Plaza de la Salud, con especial mención a las Licenciadas Anel Guzmán-Marte, Denny De La Rosa y Gilda Tolari, por sus contribuciones y disposición a suministrar datos de gran importancia para el estudio. También cabe darle mención en este apartado a la Dra. Dolores Mejía, la Dra. Jenny Cepeda y a María Fernanda Cedeño, por brindar asesoría metodológica en el desarrollo de este trabajo de investigación. Su disposición a dar su tiempo ha sido muy apreciada. Otra mención muy importante de este agra- decimiento es al Dr. Eduardo Fenocchi, luchador principal de América Latina por la prevención de este tipo de cáncer.

\section{Bibliografía}

1. Ferlay J, Ervik M, Lam F, Colombet M, Mery L, Piñeros M, Znaor A, Soerjomataram I, Bray F. Global Cancer Observatory: Cancer Today. Lyon, France: International Agency for Research on Cancer; 2018. Disponible en: https://gco.iarc. fr/today, [Citado el 01 de agosto del 2020].

2. Fecal occult blood test - Mayo Clinic [Internet]. Mayoclinic.org. 2020 Disponible en: https:// www.mayoclinic.org/tests-procedures/fecal-occult-blood-test/about/pac-20394112 [Citado el 23 de agosto del 2020].

3. Martín Álvarez I, Rodríguez Rodríguez L, García Acosta I, Hernández Morejón D, Melians Abreu SM. Sangre oculta en heces fecales: un valioso auxiliar en el diagnóstico precoz del cáncer colorrectal. Rev Ciencias Médicas [Internet]. 2016 Jun;20(3):36-41. Disponible en: http:// scielo.sld.cu/scielo.php?script=sci_arttext\&pi$\mathrm{d}=\mathrm{S} 1561-31942016000300009 \& \operatorname{lng}=$ es . [Citado el 31 de Julio 2020].

4. Fecal Immunochemical Test and Fecal Occult Blood Test | Lab Tests Online [Internet]. Labtestsonline.org. 2020. Disponible en: https:// labtestsonline.org/tests/fecal-immunochemical-test-and-fecal-occult-blood-test [Citado el 23 de agosto 2020].

5. Navarro M, Hijos G, Ramírez T, Omella I, Carrera-Lasfuentes P, Lanas Á. Fecal Hemoglobin Concentration, a Good Predictor of Risk of Advanced Colorectal Neoplasia in Symptomatic and Asymptomatic Patients. Frontiers in Medicine. 2019;6. 
6. Peńa Herrera D. (2016). Determinación de sangre oculta en heces mediante el método inmunoquímico fecal, como herramienta para diagnosticar lesiones precancerosas colorrectales en el Hospital Pablo Arturo Suárez periodo noviembre 2015 junio 2016 (Trabajo de Investigación previo a la obtención del Título de Licenciado en Laboratorio Clínico e Histotecnológico. Universidad Central del Ecuador, Quito, Ecuador). Recuperado de http:// www.dspace.uce.edu.ec/handle/25000/9277

7. Wickramasinghe D, Samaranayaka S, Lakmal C, Mathotaarachchi S, Kanishka Lal C, Keppetiyagama $\mathrm{C}$ et al. Types and Patterns of Colonic Polyps Encountered at a Tertiary Care Center in a Developing Country in South Asia. Analytical Cellular Pathology. 2014;2014:1-4.

8. Publishing H. Colon Polyps - Harvard Health [Internet]. Harvard Health. 2020 Disponible en: https://www.health.harvard.edu/a_to_z/colonpolyps-a-to-z\#:-:text=Many $\% 20$ times $\% 2 \mathrm{C} \% 20$ people\%20are\%20not,levels\%20of\%20red\%20 blood\%20cells [Citado el 19 de agosto 2020].

9. Quintero E. ¿Test químico o test inmunológico para la detección de sangre oculta en heces en el cribado del cáncer colorrectal? Gastroenterología y Hepatología. 2009;32(8):565-76.

10. Jover R, Carballo FL, Ponce M, Pérez-Riquelme F, Salas D, Cruzado-Quevedo J, et al. El test de Sangre Oculta en heces inmunológico es más coste-efectivo que el Guayaco. Comparación entre dos programas de cribado poblacional de cáncer colorrectal. Gastroenterol Hepatol. 2009;32(3):230.

11. Church J, Delaney C, Fazio V. Current therapy in colon and rectal surgery. Philadephia, Penn.: Elsevier Mosby, the Curtis Center; 2005.
12. Prueba de sangre oculta en heces [Internet]. Cancer.net. 2014. Disponible en: https://www. cancer.net/es/desplazarse-por-atencion-delcancer/diagnostico-de-cancer/pruebas-y-procedimientos/prueba-de-sangre-oculta-en-heces [Citado el 30 de julio del 2020]

13. Guittet L, Bouvier V, Mariotte N, Vallee JP, Arsène $\mathrm{D}$, Boutreux $\mathrm{S}$, et al. Comparison of a guaiac based and an immunochemical faecal occult blood test in screening for colorectal cancer in a general average risk population. Gut. 2007;56(2):210-4.

14. Fraser CG, Allison JE, Halloran SP, Young GP. A proposal to standardize reporting units for fecal immunochemical tests for hemoglobin. J Natl Cancer Inst. 2012;104(11):810-4.

15. Quintero, A. and Rigau, J., 2018. Diagnóstico y seguimiento de pacientes con sangre oculta positiva en heces fecales. [Internet] Revmedicaelectronica.sld.cu. Disponible en: http:// www.revmedicaelectronica.sld.cu/index.php/ rme/article/view/2739 [Citado el 17 de agosto 2020].

16. Gutiérrez-Stampa MA, Aguilar Gama V, Bujanda L. Utility of faecal occult blood test for the diagnosis of colorectal cancer in clinical practice in primary care. Aten Primaria. 2020;52(4):286-7.

17. del Valle Llufrio P, Romero Bareiro SR, Santana Fuentes Y. Lesiones de colon diagnosticadas por colonoscopia en pacientes con sangre oculta positiva. Rev médica electrón. 2014;36:692-9.

18. Burón A, Macià F, Andreu M, Pellisé M, Castells X, Grau J. Cribado poblacional de cáncer colorrectal: cánceres de intervalo y relación con el resultado cuantitativo del test inmunológico de sangre oculta en heces. Med Clin (Barc). 2019;152(8):303-6. 
19. Young GP, Symonds EL, Allison JE, Cole SR, Fraser CG, Halloran SP, et al. Advances in fecal occult blood tests: The FIT revolution. Dig Dis Sci. 2015;60(3):609-22.

20. Colon Polyps: Symptoms, Causes, Diagnosis, Treatment, and Prevention [Internet]. Cleveland Clinic. 2020 [cited 26 August 2020]. Available from: https://my.clevelandclinic.org/ health/diseases/15370-colon-polyps

21. Colon and Rectal Polyps | Michigan Medicine [Internet]. Uofmhealth.org. 2020 [cited 26 August 2020]. Available from: https://www. uofmhealth.org/conditions-treatments/digestive-and-liver-health/colon-and-rectal-polyps

22. Corley D, Jensen C, Marks A, Zhao W, de Boer J, Levin $\mathrm{T}$ et al. Variation of Adenoma Prevalence by Age, Sex, Race, and Colon Location in a Large Population: Implications for Screening and Quality Programs. Clinical Gastroenterology and Hepatology. 2013;11(2):172-80. 